\title{
Nonlocal Nonlinear Optical Response of Ionic Liquids under Violet Excitation
}

\author{
Cássio E. A. Santos, ${ }^{1}$ Márcio A. R. C. Alencar, ${ }^{1}$ Pedro Migowski, ${ }^{2}$ \\ Jairton Dupont, ${ }^{2}$ and Jandir M. Hickmann ${ }^{1}$ \\ ${ }^{1}$ Optics and Materials Group (OPTMA), Universidade Federal de Alagoas, P.O. Box 2051, 57061-970 Maceió, AL, Brazil \\ ${ }^{2}$ Instituto de Química, Universidade Federal do Rio Grande do Sul, 91501-970 Porto Alegre, RS, Brazil \\ Correspondence should be addressed to Márcio A. R. C. Alencar; marca@optma.org
}

Received 11 December 2012; Accepted 29 May 2013

Academic Editor: Antonia Pérez de los Ríos

Copyright (C) 2013 Cássio E. A. Santos et al. This is an open access article distributed under the Creative Commons Attribution License, which permits unrestricted use, distribution, and reproduction in any medium, provided the original work is properly cited.

\begin{abstract}
The nonlocal nonlinearity under violet excitation of ionic liquids was investigated using the Z-scan technique. The bis(trifluoromethylsulfonyl)imide $\left(\mathrm{Tf}_{2} \mathrm{~N}^{-}\right)$was employed as anionic part, while the cationic part consisted of four different imidazolium $\left(\mathrm{C}_{n} \mathrm{MI}\right.$, with $n=4,6,8$, and 10) derivatives. The thermooptical coefficients, nonlinear refractive indexes of thermal origin, and degree of nonlocality were obtained for laser excitation tuned at $410 \mathrm{~nm}$. Our results indicate that ionic liquids can be exploited as very efficient nonlinear media with large nonlocal character under violet excitation.
\end{abstract}

\section{Introduction}

Ionic liquids (ILs) have attracted a large amount of research interest in recent years due to their peculiar chemical and physical properties [1-7]. In particular, it has been demonstrated that ILs present large thermooptical responses in the infrared and blue region of the electromagnetic spectra [810]. Owing to this fact, ILs can be exploited in the investigation of nonlocal optical phenomena $[8,9]$ and for thermallens measurements as a solvent [10]. Moreover, some of the ILs' physical and chemical properties are strongly dependent of the ions features that compose them [9, 11-13]. This suggests that materials with improved physical properties, such as nonlocal nonlinearities, might be engineered by a suitable choice of ILs' anionic and cationic parts.

A nonlinear optical effect can be classified as nonlocal if the light induced response at a certain point of the material depends also on the values of the electromagnetic field at surrounding regions [14]. Materials of different natures can exhibit nonlocal nonlinearities due to specific physical mechanisms [15-21]. For instance, organic compounds, which present large thermooptical responses, have been currently used in the investigation of optical nonlocal effects [2226]. In fact, these media possess a large refractive nonlocal response owing to the change on the refractive index as a consequence of the heat conduction process.

New effects, such as suppression of the modulational instability [27, 28], nonlinear shock waves [29-31], stabilization of complex soliton structures [32-35], and prevention of the multidimensional beams' collapse $[36,37]$ have been observed exploiting the peculiar features of this kind of nonlinearity. However, there is a huge number of new nonlinear effects with nonlocal character that have been poorly investigated or remain unexploited up to this moment. This limitation can be partially attributed to the lack of known nonlocal media with well-characterized properties suitable to this end. Owing to this fact, there is still a great interest in the development of new materials with improved nonlocal nonlinear responses.

In this work, we investigate the influence of the cationic part on the nonlocal optical nonlinearities of ILs under violet excitation. Four different compositions of ILs were investigated, using bis(trifluoromethylsulfonyl)imide $\left(\mathrm{Tf}_{2} \mathrm{~N}^{-}\right)$as anionic part, while the cationic part was composed of different derivatives of imidazolium $\left(\mathrm{C}_{n} \mathrm{MI}\right.$, with $n=4,6,8$, and 10). Their thermo-optical properties and nonlocal nonlinear responses were measured using the Z-scan technique [38, 39]. We observed that under violet excitation large refractive 
nonlinear responses and nonlocal degree of nonlocality are achieved. Our results indicate that those organic materials can be engineered aiming at the development of more efficient nonlinear media with nonlocal character in this wavelength region.

\section{Nonlinear Optics and Nonlocality}

Although the propagation of an optical beam through a nonlinear medium has been intensively investigated along the last decades, the number of phenomena that can be associated with the beam-medium interaction never stops increasing as well as the development of applications exploiting them. In this sense, nonlocal effects correspond just to a family of nonlinear optical phenomenon and can be described, therefore, by the same physical and mathematical main principles.

Hence, let us assume the propagation, along the $z$ axis, of a monochromatic and linearly polarized optical beam through an isotropic and homogeneous medium. The scalar amplitude of this beam's electrical field is given by

$$
E(x, y, z, t)=\left(\frac{2}{c \varepsilon_{0} n}\right)^{1 / 2} A(x, y) \exp (i k z-i \omega t),
$$

where $A(x, y)$ is the slowly varying envelope amplitude and $k=n k_{0}=n(\omega / c)$ is the modulus of the wavevector. This beam propagation is ruled by the paraxial wave equation for the envelope

$$
i \frac{\partial A}{\partial z}+\frac{1}{2 k}\left(\frac{\partial^{2} A}{\partial x^{2}}+\frac{\partial^{2} A}{\partial y^{2}}\right)+k_{0} \Delta n A=-i \frac{\alpha_{0}}{2} A,
$$

where $\alpha_{0}$ is the medium linear absorption and $\Delta n$ is the material's refractive index change induced optically, in other words, the term of the nonlinear interaction. For a local Kerr nonlinearity, this nonlinear term is just the product of the material nonlinear refractive index by the field intensity and (2) can describe this beam-medium interaction. On the other hand, if the nonlinearity has a nonlocal character, $\Delta n$ is no longer this former simple product, but a coupling term between (2) and an equation that rules the nonlocal modifications of the material's optical properties. In particular, for thermal nonlinearities

$$
\Delta n=\left(\frac{d n}{d T}\right) \Delta T
$$

where $d n / d T$ is the thermooptical coefficient and $\Delta T$ is the local temperature change owing to the material's light absorption.

If we neglect the longitudinal changes on the temperature field, $\Delta T$ can be represented by a two-dimensional function and the second equation that rules the nonlocal interaction is the $2 \mathrm{D}$ heat equation

$$
\left(\frac{\partial^{2}}{\partial x^{2}}+\frac{\partial^{2}}{\partial y^{2}}\right) \Delta T-C \Delta T=-\frac{\alpha_{0}}{\rho_{0} c_{p} D}|A|^{2},
$$

where $c_{p}, \rho_{0}$, and $D$ are the medium heat capacity, density, and heat diffusivity, respectively. It was shown by Ghofraniha

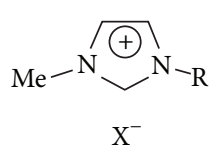

$$
\begin{aligned}
& \text { BMI.NTf } 2, R={ }^{n} \mathrm{Bu}, \mathrm{X}=\mathrm{N}\left(\mathrm{SO}_{2} \mathrm{CF}_{3}\right)_{2} \\
& \text { HMI.NTf } 2, R={ }^{n} \mathrm{Hex}, \mathrm{X}=\mathrm{N}\left(\mathrm{SO}_{2} \mathrm{CF}_{3}\right)_{2} \\
& \text { OMI.NTf } 2, R={ }^{n} \text { Oct, } \mathrm{X}=\mathrm{N}\left(\mathrm{SO}_{2} \mathrm{CF}_{3}\right)_{2} \\
& \text { DMI.NTf } 2, R={ }^{n} \text { Dec, } \mathrm{X}=\mathrm{N}\left(\mathrm{SO}_{2} \mathrm{CF}_{3}\right)_{2}
\end{aligned}
$$

FIgURE 1: Schematic representation of ILs structures.

et al. [30] that the parameter $C=1 / w_{0}^{2} \sigma^{2}$ is a constant that depends on the beam minimum waist, $w_{0}$, and the degree of nonlocality of the medium, $\sigma^{2}$. The larger the value of $\sigma$, the more nonlocal is the medium. On the other hand, if $\sigma=0$, the medium can only present a local nonlinearity. Moreover, they also demonstrated that this quantity is indeed related to the material's thermal and optical properties by

$$
\sigma=\sqrt{\frac{\kappa\left|n_{2}\right|}{\alpha_{0}|d n / d T| w_{0}^{2}}}
$$

where $\kappa$ is the heat conductivity and $n_{2}$ is the nonlinear refractive of the material.

It is worth mentioning that (4) represents a special case of optical nonlocality, the thermal-lens effect. Different physical mechanisms can also generate nonlocal effects [15-21], which means that the medium nonlocal response can be given in different ways. However, this formalism, proposed in [30], has a huge practical impact, as it allowed us to connect the degree of nonlocality with the nonlinear properties of the medium.

\section{Experiment}

In Figure 1, the schematic molecular structures of the investigated materials are presented. In this work, we employed $\mathrm{Tf}_{2} \mathrm{~N}^{-}$as the anion, while the cation was modified, by changing the number of carbons present in the radical $R^{2}$, namely, 4 (1-butyl-3-methylimidazolium-BMI ${ }^{+}$), 6 (1-hexyl3-methylimidazolium- $\mathrm{HMI}^{+}$), 8 (1-octyl-3-methylimidazolium- $\mathrm{OMI}^{+}$), and 10 (1-decyl-3-methylimidazolium$\left.\mathrm{DMI}^{+}\right)$. These ionic liquids were prepared as reported previously $[40,41]$.

The Z-scan measurements were performed using the second harmonic of a mode-locked Ti:Sapphire laser (200 fs, $76 \mathrm{MHz}$ ) tuned at $820 \mathrm{~nm}$. This linearly polarized violet beam, tuned at $410 \mathrm{~nm}$, was then focused onto the sample by convergent lens of $7.5 \mathrm{~cm}$ focal length. The ILs were contained in a $1 \mathrm{~mm}$ width quartz cell and placed on a translation stage. The cell position was then modified around the lens focal plane $(z=0)$ by a computer controlled stepping motor. The light transmitted through the cell was spatially filtered by a small aperture and its intensity measured by a photodetector as a function of the sample position. The detected signal was amplified by a lock-in amplifier and then processed by a computer. Nonlinear absorption measurements were performed with the same experimental setup but using a configuration without aperture.

Assuming a material that does not exhibit nonlinear absorption, for high repetition rate pulsed excitations, the 
thermal contribution for the transmittance in Z-scan experiments can be obtained by [39]

$$
\operatorname{Tr}(x)=1+\theta \operatorname{Tan}^{-1}\left[\frac{2 x}{\left(9+x^{2}\right)\left(1+x^{2}\right) / 2 \tau+\left(3+x^{2}\right)}\right],
$$

where $\theta$ is the thermal induced phase-shift, $x=z / z_{0}$ and $\tau=$ $t / t_{c_{0}}$ are the normalized distance and time, respectively, and $t_{c_{0}}=w_{0}^{2} / 4 D$ corresponds to the characteristic thermal lens time constant. For measurements performed at a fixed time $t$, one can use (6) to fit the experimental results and evaluate the values of $\theta$. In the absence of fluorescence, the thermooptical coefficient is given by [39]

$$
\frac{d n}{d T}=\frac{\lambda \kappa}{P \alpha_{0} L_{\mathrm{eff}}} \theta
$$

where $P$ is the average laser power, and $L_{\text {eff }}=\left(1-e^{-\alpha_{0} L}\right) / \alpha_{0}$ is the sample effective length.

The thermal contribution for the nonlinear refractive index is obtained from the difference between the maximum and minimum transmittance measured in the Z-scan measurements [38]

$$
n_{2}=\frac{\sqrt{2} \Delta \operatorname{Tr}_{p v}}{0,406(1-S)^{0,25} k L_{\text {eff }} I_{0}},
$$

where $S$ is the aperture transmittance, $k$ is the modulus of the beam wave vector, and $I_{0}$ is the maximum laser intensity. We can express this change on the refractive index in terms of an effective nonlinear refractive index coefficient $n_{2}$ by the relation [42]

$$
\Delta n=n_{2} I_{0},
$$

where $I_{0}$ is the maximum laser intensity.

Although for thermal contributions to the optical nonlinearity the definition of a nonlinear refractive index is not accurate, the definition of this quantity permits a convenient comparison between the thermally induced nonlinear refraction with others of nonthermal origin. Moreover, as it was stated in Section 2, employing this definition, it is possible to obtain the degree of nonlocality.

\section{Results and Discussion}

The transmittance spectra of the investigated ILs are presented in Figure 2. As can be observed, all compounds present a large value of transmittance (larger than 75\%) in the visible, but present an absorption increase in the violet and ultraviolet spectral regions. As this change on the absorption spectrum is usually associated with an increase on the nonlinear refraction response of the medium, the values of $d n / d T, n_{2}$, and $\sigma$ could also be modified, exhibiting a dispersion-like behavior. Owing to this fact, the suitable choice of the light wavelength may lead to an improved nonlocal response of a medium. This is the main reason for the choice of the violet excitation employed in this work.

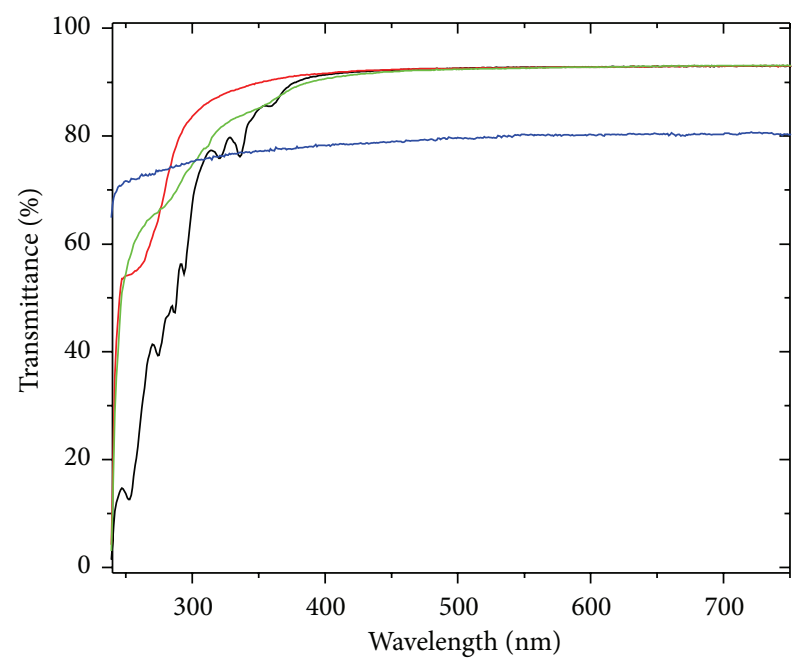

FIGURE 2: Transmittance spectra of the investigated ILs: BMI-NTf ${ }_{2}$ (black), HMI-NTf 2 (red), OMI·NTf (green), and DMI-NTf (blue).

In Figure 3, the results for closed aperture Z-scan measurement of all the samples are shown. As can be observed, these ILs present larges negative nonlinear responses of thermal origin. Fitting these results with (1), and using the reported values for the heat capacity [43] as well as (2), (3), and (4), the thermooptical coefficient $(d n / d T)$ and the thermal nonlinear refractive index $\left(n_{2}\right)$ of these ILs were obtained. From these results, the degree of nonlocality of all investigated compounds was also obtained from (5). It is worth mentioning that the open aperture experiment did not reveal a measurable nonlinear absorption. A summary of these results is presented in Table 1.

Z-scan measurements employing the same femtosecond violet beam but with a reduced repetition rate were also performed. In this case, using a pulse-picker, the repetition rate was reduced into the range of $100 \mathrm{~Hz}$ to $1 \mathrm{kHz}$ and the laser peak power varied between 0.5 and $1 \mathrm{~kW}$, which was even larger than the peak power employed at the high repetition rate experiments. However, the characteristic Z-scan curves signature were absent for all experimental conditions exploited in this work. This result indicates that the electronic (ultrafast) contribution to the nonlinear refractive index of these liquids is much smaller than the thermal part and did not affect significantly the results displayed in Table 1.

As can be observed, all samples presented large values of $n_{2}, d n / d T$, and $\sigma$. It should be noticed that although the OMI-NTf ${ }_{2}$ compound possesses the largest values of nonlinear refractive index and thermooptical coefficient, its degree of nonlocality is approximately equal to the other ILs when the experimental error is taken into account. This corroborates with results obtained for the same ILs but excited in the near infrared region [9]. Therefore, for infrared or violet excitation, the change on the cationic part does not modify significantly the ILs nonlocal response.

Additionally, it was also verified that although $n_{2}$ values are approximately two orders of magnitude larger, in modulus, for light in the violet than in the infrared region [9], 


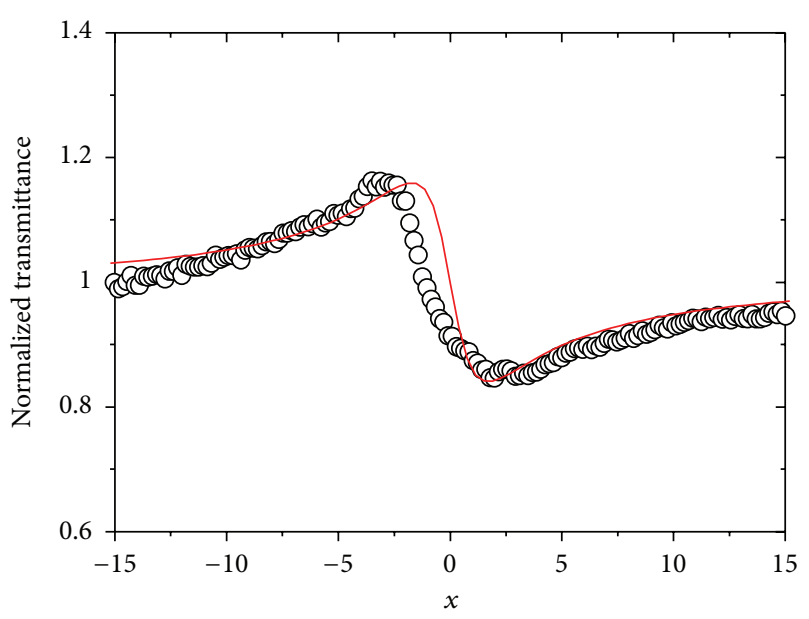

(a)

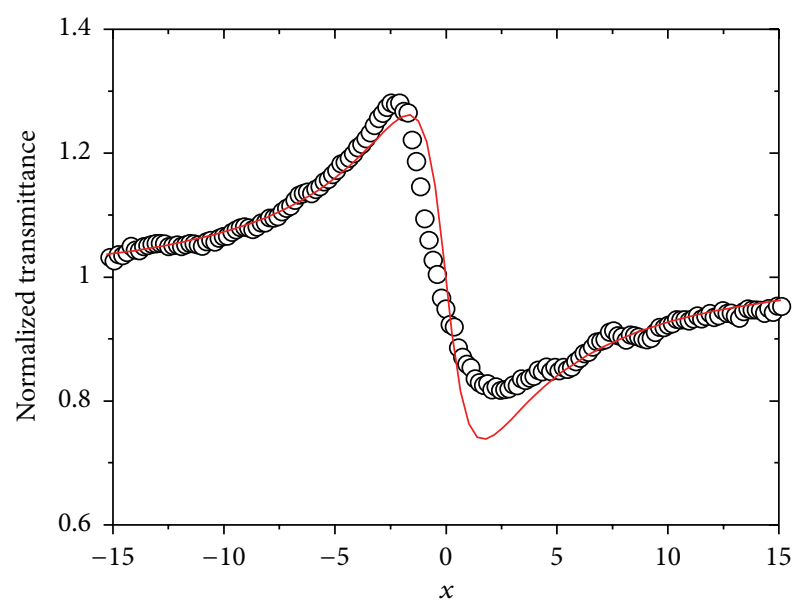

(c)

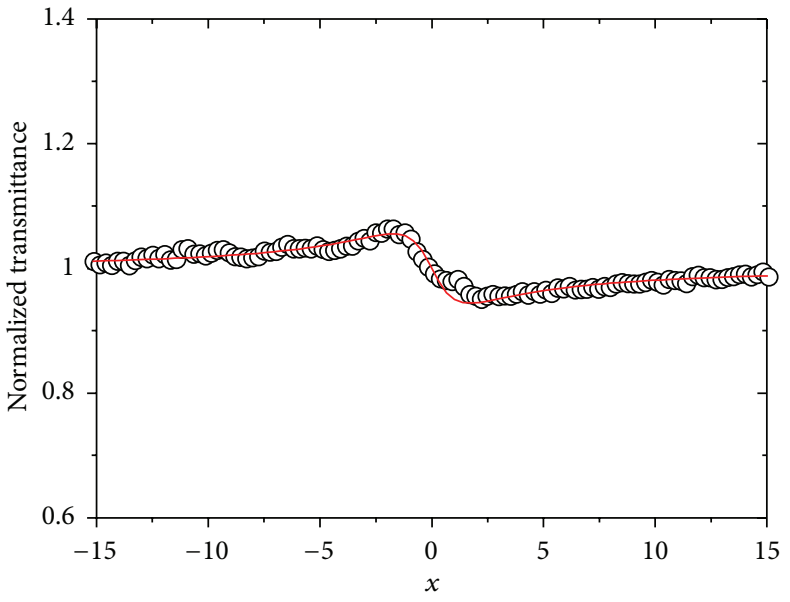

(b)

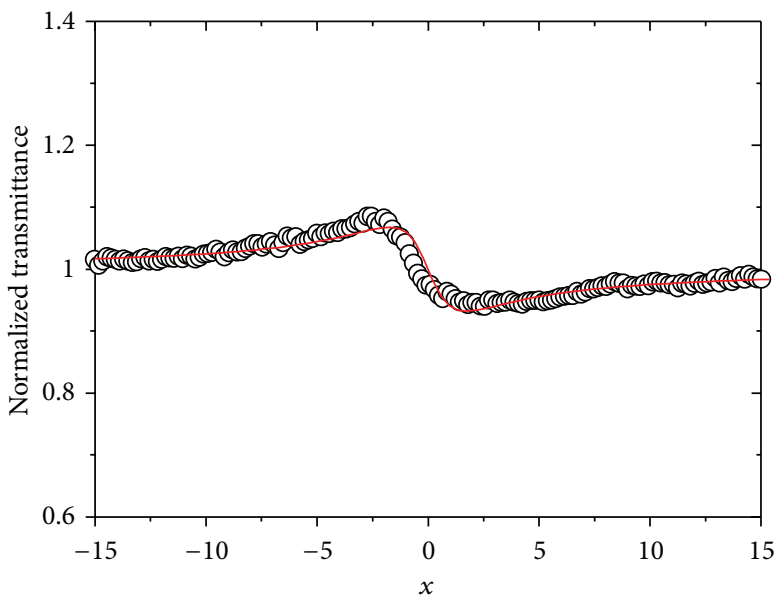

(d)

Figure 3: Z-scan curves for ILs composed by $\mathrm{Tf}_{2} \mathrm{~N}^{-}$and (a) $\mathrm{BMI}^{+}$measured with $P_{\text {LASER }}=9.24 \mathrm{~mW}$, (b) $\mathrm{HMI}^{+}$with $P_{\text {LASER }}=9.01 \mathrm{~mW}$, (c) $\mathrm{OMI}^{+}$with $P_{\text {LASER }}=8.77 \mathrm{~mW}$, and (d) $\mathrm{DMI}^{+}$with $P_{\text {LASER }}=8.65 \mathrm{~mW}$. The minimum beam waist was $w_{0}=18 \mu \mathrm{m}$ and the aperture transmittance $S=0.002$ for all measurements. White dots correspond to the experimental data and the red curves are the fittings using (6).

TABLE 1: Experimental linear absorption coefficient $\left(\alpha_{0}\right)$, heat conductivity $(\kappa)$, thermal nonlinear refractive index $\left(n_{2}\right)$, thermo-optical coefficient $(d n / d T)$, and degree of nonlocality $(\sigma)$ of the investigated ionic liquids.

\begin{tabular}{lccccc}
\hline Sample & $\alpha_{0}\left(\mathrm{~cm}^{-1}\right)$ & $\kappa(\mathrm{W} / \mathrm{m} \mathrm{K})[43]$ & $n_{2}\left(\mathrm{~cm}^{2} / \mathrm{W}\right)$ & $d n / d T\left(\mathrm{~K}^{-1}\right)$ & $\sigma$ \\
\hline BMI-Tf $_{2} \mathrm{~N}$ & 0.161 & 0.128 & $(-3.9 \pm 1.2) 10^{-8}$ & $(-9.6 \pm 2.9) 10^{-5}$ & $(0.96 \pm 0.38)$ \\
$\mathrm{HMI}_{-}{ }_{2} \mathrm{~N}$ & 0.071 & 0.127 & $(-1.9 \pm 0.6) 10^{-8}$ & $(-9.3 \pm 2.8) 10^{-5}$ & $(1.06 \pm 0.43)$ \\
$\mathrm{OMI}_{-} \mathrm{Tf}$ & $0.12 \mathrm{~N}$ & 0.128 & $(-5.1 \pm 1.5) 10^{-8}$ & $(-12.2 \pm 3.7) 10^{-5}$ & $(0.83 \pm 0.33)$ \\
DMI-Tf $_{2} \mathrm{~N}$ & 0.319 & 0.132 & $(-1.8 \pm 0.5) 10^{-8}$ & $(-2.5 \pm 0.8) 10^{-5}$ & $(0.97 \pm 0.39)$ \\
\hline
\end{tabular}

the values of $d n / d T$ and $\sigma$ are only slightly modified if you take into account the experimental uncertainties. This result suggests that even among materials of the same kind, ILs, for instance, a structural modification that enhances the thermal nonlinear refractive index of a material does not imply on a correspondent change of the medium nonlocality.

Of course, there is a clear indication that for smaller excitation wavelengths, the degree of nonlocality appears to be larger when compared with the infrared response. Unfortunately, the experimental errors involved did not allow us to achieve more accurate results. There is still a strong need of novel methods aiming at the measurement of the degree of nonlocality parameter with smaller uncertainties.

\section{Conclusions}

In summary, we investigated the nonlinear optical response with nonlocal character of four different ILs compounds under violet excitation. Using the Z-scan technique, large values for the ILs thermooptical coefficient, thermal nonlinear 
refractive index and the degree of nonlocality were obtained. It was observed that the structural change due to the modification of the cationic part does not modify significantly the ILs nonlocal response. Moreover, comparing the results obtained using violet and infrared [9] excitation, it was verified that although the $n_{2}$ values are approximately two orders of magnitude larger for the smaller light wavelength, the degree of nonlocality and the thermooptical coefficient are slightly modified by this change, which indicates that the nonlocal nonlinear refractive index is not the only parameter that rules the behavior of a nonlinear material's degree of nonlocality. Our results confirm that ILs are very promising media for nonlinear optical applications with nonlocal character.

\section{Acknowledgments}

The authors would like to thank the financial support from FAPEAL, Pronex/FAPEAL, CNPq/MCT, CAPES Pró-equipamentos/PROCAD/PROCAD-NF, Nanofoton Network, and INCT-FOTONICOM. They also would like to thank M. G. da Silva and M. R. Meneghetti for the help with the cleaning procedures of the cells used in the experiments.

\section{References}

[1] J. Dupont, R. F. de Souza, and P. A. Z. Suarez, "Ionic liquid (molten salt) phase organometallic catalysis," Chemical Reviews, vol. 102, no. 10, pp. 3667-3692, 2002.

[2] S. Ono, S. Seki, R. Hirahara, Y. Tominari, and J. Takeya, "High-mobility, low-power, and fast-switching organic fieldeffect transistors with ionic liquids," Applied Physics Letters, vol. 92, no. 10, Article ID 103313, 3 pages, 2008.

[3] C. E. Song, "Enantioselective chemo- and bio-catalysis in ionic liquids," Chemical Communications, no. 9, pp. 1033-1043, 2004.

[4] T. Rüther, T. D. Huynh, J. Huang et al., "Correction to stable cycling of lithium batteries using novel boronium-cation-based ionic liquid electrolytes," Chemistry of Materials, vol. 22, no. 3, pp. 1038-1045, 2010.

[5] H. S. Park, B. G. Choi, S. H. Yang et al., "Ionic-liquid-assisted sonochemical synthesis of carbon-nanotube-based nanohybrids: control in the structures and interfacial characteristics," Small, vol. 5, no. 15, pp. 1754-1760, 2009.

[6] J. Dupont, G. S. Fonseca, A. P. Umpierre, P. F. P. Fichtner, and S. R. Teixeira, "Transition-metal nanoparticles in imidazolium ionic liquids: recycable catalysts for biphasic hydrogenation reactions," Journal of the American Chemical Society, vol. 124, no. 16, pp. 4228-4229, 2002.

[7] C. J. Bowlas, D. W. Bruce, and K. R. Seddon, "Liquid-crystalline ionic liquids," Chemical Communications, no. 14, pp. 1625-1626, 1996.

[8] R. F. Souza, M. A. R. C. Alencar, M. R. Meneghetti, J. Dupont, and J. M. Hickmann, "Nonlocal optical nonlinearity of ionic liquids," Journal of Physics Condensed Matter, vol. 20, no. 15, Article ID 155102, 2008.

[9] C. E. A. Santos, M. A. R. C. Alencar, P. Migowski, J. Dupont, and J. M. Hickmann, "Anionic and cationic influence on the nonlocal nonlinear optical response of ionic liquids," Chemical Physics, vol. 403, pp. 33-36, 2012.
[10] C. D. Tran, S. Challa, and M. Franko, "Ionic liquids as an attractive alternative solvent for thermal lens measurements," Analytical Chemistry, vol. 77, no. 22, pp. 7442-7447, 2005.

[11] H. Tokuda, K. Hayamizu, K. Ishii, M. A. B. H. Susan, and M. Watanabe, "Physicochemical properties and structures of room temperature ionic liquids. 2. variation of alkyl chain length in imidazolium cation," Journal of Physical Chemistry B, vol. 109, no. 13, pp. 6103-6110, 2005.

[12] C. S. Consorti, P. A. Z. Suarez, R. F. de Souza et al., "Identification of 1,3-dialkylimidazoIium salt supramolecular aggregates in solution," Journal of Physical Chemistry B, vol. 109, no. 10, pp. 4341-4349, 2005.

[13] A. Triolo, O. Russina, B. Fazio, R. Triolo, and E. Di Cola, "Morphology of 1-alkyl-3-methylimidazolium hexafluorophosphate room temperature ionic liquids," Chemical Physics Letters, vol. 457, no. 4-6, pp. 362-365, 2008.

[14] W. Królikowski, O. Bang, N. I. Nikolov et al., "Modulational instability, solitons and beam propagation in spatially nonlocal nonlinear media," Journal of Optics B, vol. 6, no. 5, pp. S288S294, 2004.

[15] A. V. Mamaev, A. A. Zozulya, V. K. Mezentsev, D. Z. Anderson, and M. Saffman, "Bound dipole solitary solutions in anisotropic nonlocal self-focusing media," Physical Review A, vol. 56, no. 2, pp. R1110-R1113, 1997.

[16] A. V. Mamaev, M. Saffman, and A. A. Zozulya, "Phasedependent collisions of $(2+1)$-dimensional spatial solitons," Journal of the Optical Society of America B, vol. 15, no. 7, pp. 2079-2082, 1998.

[17] A. A. Zozulya, D. Z. Anderson, A. V. Mamaev, and M. Saffman, "Self-focusing and soliton formation in media with anisotropic nonlocal material response," Europhysics Letters, vol. 36, no. 6, pp. 419-424, 1996.

[18] P. D. Rasmussen, O. Bang, and W. Krolikowski, "Theory of nonlocal soliton interaction in nematic liquid crystals," Physical Review E, vol. 72, no. 6, Article ID 066611, 7 pages, 2005.

[19] M. Peccianti, C. Conti, G. Assanto, A. de Luca, and C. Umeton, "Nonlocal optical propagation in nonlinear nematic liquid crystals," Journal of Nonlinear Optical Physics and Materials, vol. 12, no. 4, pp. 525-538, 2003.

[20] A. A. Minzoni, N. F. Smyth, Z. Y. Xu, and Y. S. Kivshar, "Stabilization of vortex-soliton beams in nematic liquid crystals," Physical Review A, vol. 79, no. 6, Article ID 063808, 7 pages, 2009.

[21] G. Assanto, N. F. Smyth, and A. L. Worthy, "Two-color, nonlocal vector solitary waves with angular momentum in nematic liquid crystals," Physical Review A, vol. 78, no. 1, Article ID 013832, 8 pages, 2008.

[22] S. Skupin, O. Bang, D. Edmundson, and W. Krolikowski, "Stability of two-dimensional spatial solitons in nonlocal nonlinear media," Physical Review E, vol. 73, no. 6, Article ID 066603, 8 pages, 2006.

[23] C. Rotschild, O. Cohen, O. Manela, M. Segev, and T. Carmon, "Solitons in nonlinear media with an infinite range of nonlocality: first observation of coherent elliptic solitons and of vortexring solitons," Physical Review Letters, vol. 95, no. 21, Article ID 213904, 4 pages, 2005.

[24] A. Minovich, D. N. Neshev, A. Dreischuh, W. Krolikowski, and Y. S. Kivshar, "Experimental reconstruction of nonlocal response of thermal nonlinear optical media," Optics Letters, vol. 32, no. 12, pp. 1599-1601, 2007. 
[25] R. Fischer, D. N. Neshev, W. Krolikowski et al., "Oblique interaction of spatial dark-soliton stripes in nonlocal media," Optics Letters, vol. 31, no. 20, pp. 3010-3012, 2006.

[26] D. Buccoliero, A. S. Desyatnikov, W. Krolikowski, and Y. S. Kivshar, "Boundary effects on the dynamics of higher-order optical spatial solitons in nonlocal thermal media," Journal of Optics A, vol. 11, no. 9, Article ID 094014, 2009.

[27] B. K. Esbensen, A. Wlotzka, M. Bache, O. Bang, and W. Krolikowski, "Modulational instability and solitons in nonlocal media with competing nonlinearities," Physical Review A, vol. 84, no. 5, Article ID 053854, 2011.

[28] M. Peccianti, C. Conti, and G. Assanto, "Optical modulational instability in a nonlocal medium," Physical Review E, vol. 68, no. 2, Article ID 025692, 4 pages, 2003.

[29] W. J. Wan, S. Jia, and J. W. Fleischer, "Dispersive superfluid-like shock waves in nonlinear optics," Nature Physics, vol. 3, no. 1, pp. 46-51, 2007.

[30] N. Ghofraniha, C. Conti, G. Ruocco, and S. Trillo, "Shocks in nonlocal media," Physical Review Letters, vol. 99, no. 4, Article ID 043903, 4 pages, 2007.

[31] N. Ghofraniha, L. S. Amato, V. Folli, S. Trillo, E. DelRe, and C. Conti, "Measurement of scaling laws for shock waves in thermal nonlocal media," Optics Letters, vol. 37, no. 12, pp. 2325-2327, 2012.

[32] B. Alfassi, C. Rotschild, O. Manela, M. Segev, and D. N. Christodoulides, "Nonlocal surface-wave solitons," Physical Review Letters, vol. 98, no. 21, Article ID 213901, 4 pages, 2007.

[33] B. Alfassi, C. Rotschild, and M. Segev, "Incoherent surface solitons in effectively instantaneous nonlocal nonlinear media," Physical Review A, vol. 80, no. 4, Article ID 041808, 4 pages, 2009.

[34] P. D. Rasmussen, F. H. Bennet, D. N. Neshev et al., "Observation of two-dimensional nonlocal gap solitons," Optics Letters, vol. 34, no. 3, pp. 295-297, 2009.

[35] F. Maucher, W. Krolikowski, and S. Skupin, "Stability of solitary waves in random nonlocal nonlinear media," Physical Review A, vol. 85, no. 6, Article ID 063803, 11 pages, 2012.

[36] D. Neshev, G. McCarthy, W. Krolikowski et al., "Dipole-mode vector solitons in anisotropic nonlocal self-focusing media," Optics Letters, vol. 26, no. 15, pp. 1185-1187, 2001.

[37] W. Krolikowski, O. Bang, and J. Wyller, "Nonlocal incoherent solitons," Physical Review E, vol. 70, no. 3, Article ID 036617, 5 pages, 2004.

[38] M. Sheik-Bahae, A. A. Said, T. H. Wei, D. J. Hagan, and E. W. van Stryland, "Sensitive measurement of optical nonlinearities using a single beam," IEEE Journal of Quantum Electronics, vol. 26, no. 4, pp. 760-769, 1990.

[39] M. Falconieri, "Thermo-optical effects in Z-scan measurements using high-repetition-rate lasers," Journal of Optics A, vol. 1, no. 6, article 662, 1999.

[40] P. Migowski, G. Machado, S. R. Texeira et al., "Synthesis and characterization of nickel nanoparticles dispersed in imidazolium ionic liquids," Physical Chemistry Chemical Physics, vol. 9, no. 34, pp. 4814-4821, 2007.

[41] P. A. Z. Suarez, J. E. L. Dullius, S. Einloft, R. F. de Souza, and J. Dupont, "The use of new ionic liquids in two-phase catalytic hydrogenation reaction by rhodium complexes," Polyhedron, vol. 15, no. 7, pp. 1217-1219, 1996.

[42] R. W. Boyd, Nonlinear Optics, Academic Press, San Diego, Calif, USA, 2nd edition, 2003.
[43] R. Ge, C. Hardacre, P. Nancarrow, and D. W. Rooney, “Thermal conductivities of ionic liquids over the temperature range from $293 \mathrm{~K}$ to $353 \mathrm{~K}$," Journal of Chemical and Engineering Data, vol. 52, no. 5, pp. 1819-1823, 2007. 

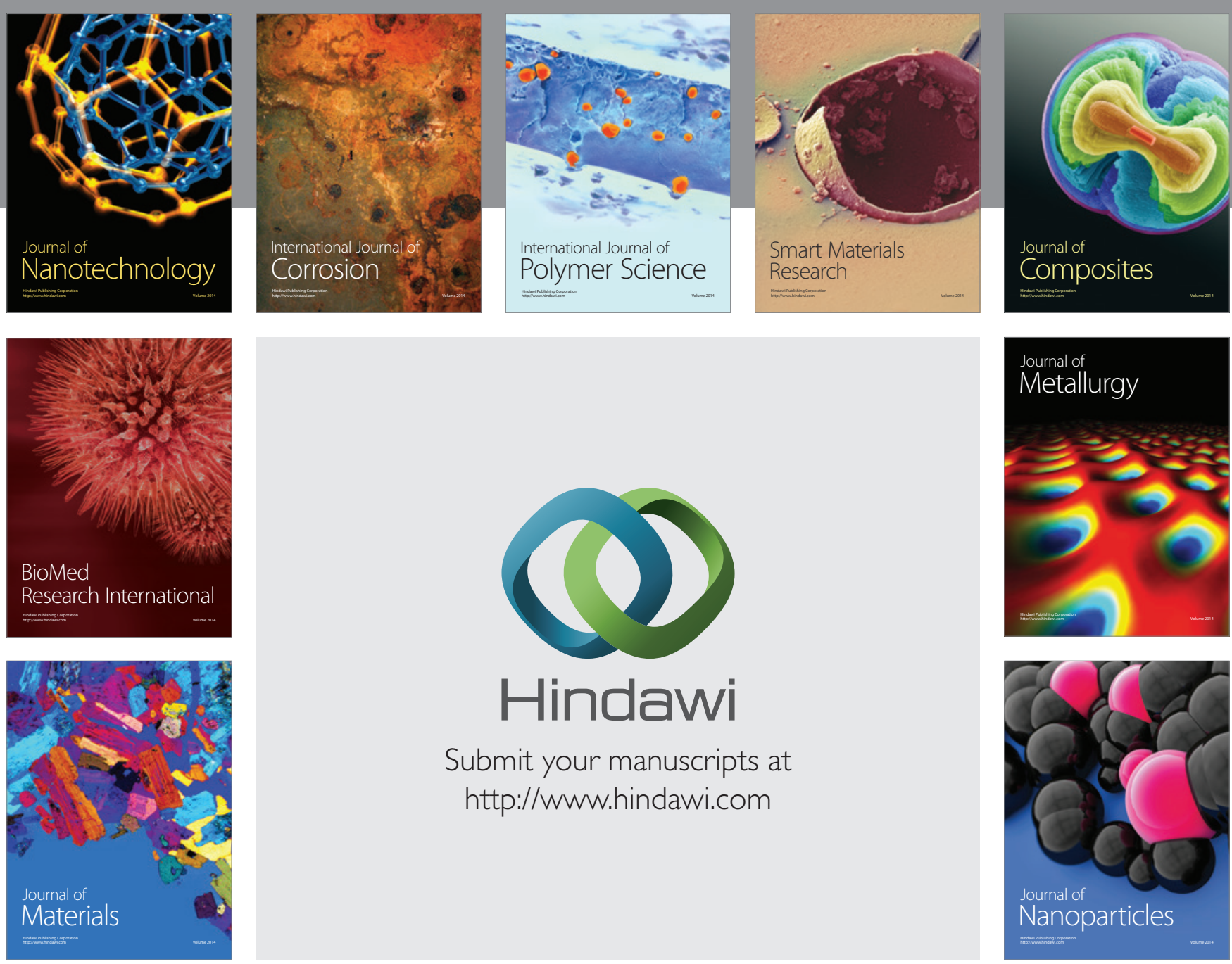

Submit your manuscripts at http://www.hindawi.com
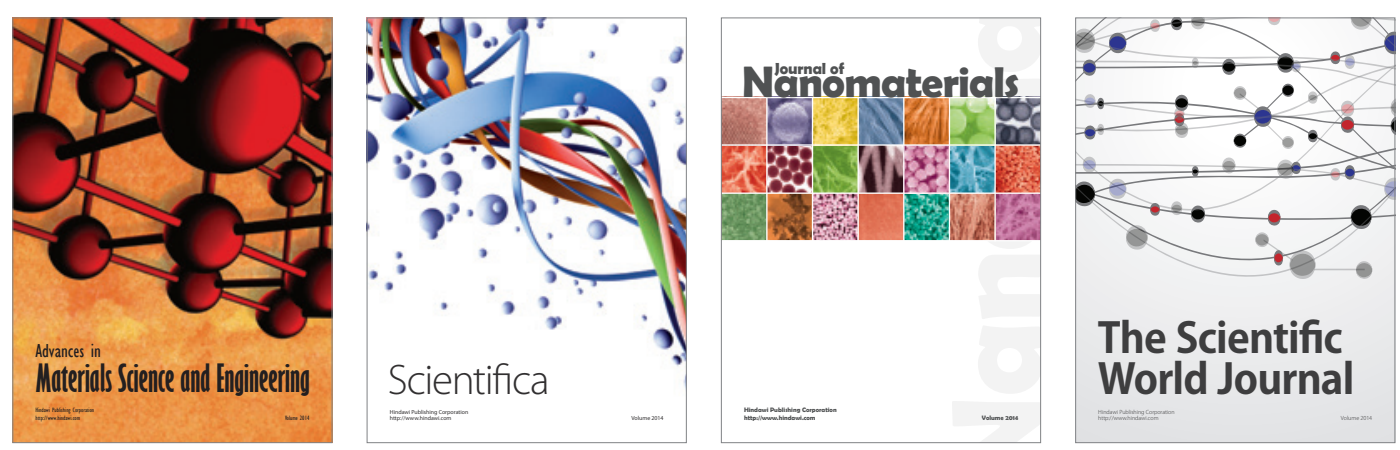

\section{The Scientific World Journal}
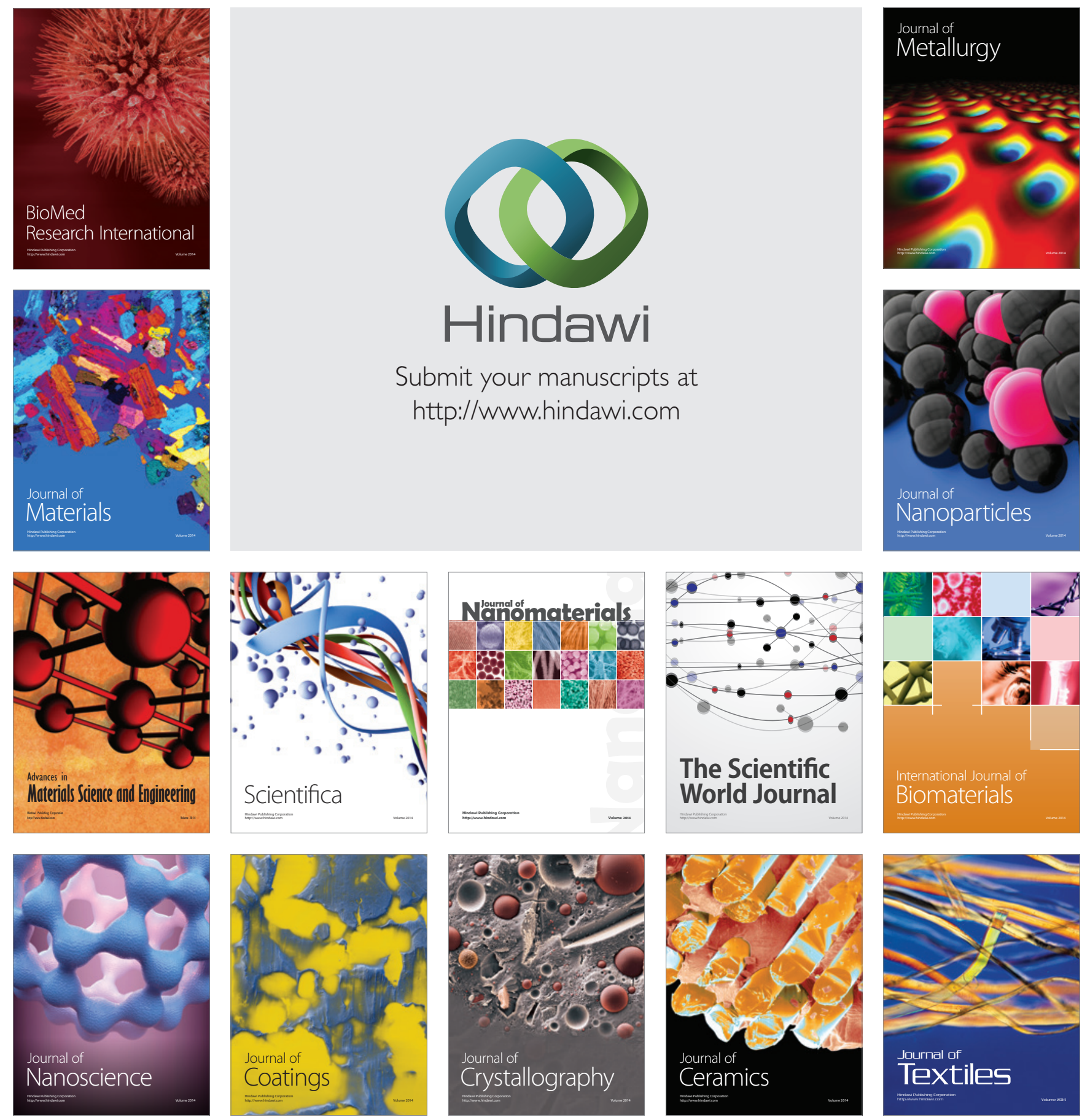